\title{
LA RESPONSABILIDAD SOCIAL \\ EMPRESARIAL FRENTE A LOS \\ BENEFICIOS TRIBUTARIOS \\ Y LA IMAGEN CORPORATIVA
}

CORPORATE SOCIAL RESPONSIBILITY VERSUS TAX BENEFITS

AND CORPORATE BRAND

Liliana LuQue SÁNChez ${ }^{1}$

Natalia Luque ${ }^{2}$

July Villalba ${ }^{3}$

1 Maestría en educación, Corporación Universitaria Iberoamericana. Bogotá, Colombia. Correo electrónico: liluquesa@gmail.com

Orcid: https://orcid.org/0000-0002-2467-7871

2 Bióloga, Universidad Nacional de Colombia. Bogotá, Colombia. Correo electrónico: nluques@unal.edu.co

Orcid: https://orcid.org/0000-0002-7127-2711

3 Investigadora auxiliar, Corporación Universitaria Iberoamericana. Bogotá, Colombia. Correo electrónico: july.villalba@ibero.edu.co; villalbajuly51@gmail.com

Orcid: https://orcid.org/0000-0002-2359-1216

Código JeL: M14, Q56

Fecha de recepción: 19/12/2019

Fecha de aceptación: 27/04/2020

DOI: https://doi.org/10.18601/16577175.n27.08 


\section{Resumen}

La implementación de programas de responsabilidad social empresarial (RSE) puede otorgar a las empresas valores agregados como la fidelización de los diferentes grupos de interés, así como aportes sociales, ambientales, organizacionales y tributarios importantes.

El presente estudio se enfocó en establecer el conocimiento que el personal del área administrativa o contable tiene sobre los beneficios tributarios a los que pueden acceder las empresas al implementar un programa de RSE. El proyecto se realizó a partir de un estudio de tipo descriptivo retrospectivo, con datos cuantitativos y cualitativos obtenidos a partir de encuestas, mostrando falencias importantes en el conocimiento del tema, así como conceptos muy generales por parte de los profesionales de las áreas encuestadas.

Palabras clave: beneficios tributarios; gestión de stakeholders; imagen corporativa; mercadeo; RSE.

\section{Abstract}

The implementation of corporate social responsibility (CSR) programs can give added value to companies such as building customer loyalty of the different stakeholders, as well as important social, environmental, organizational and tax contributions.

The present study focused on establishing the knowledge that the administrative and/or accounting personnel have regarding the tax benefits that companies can access when implementing a CSR program. The project was carried out from a retrospective descriptive study perspective, with quantitative and qualitative data obtained from surveys, showing important flaws in the knowledge regarding the tax benefits and CSR concepts in general by professionals in the surveyed areas.

Keywords: corporate image; CSR; marketing; stakeholders management; tributary benefits.

\section{INTRODUCCIÓN}

Actualmente las dinámicas del mercado han cambiado y se rigen no solo por la oferta y la demanda; las decisiones de compra o uso de servicios están marcadas también por el nivel de veeduría que realizan los consumidores y los grupos de interés o stakeholders, tanto internos como externos, basados en el conocimiento del comportamiento de las organizaciones, sus proceso productivos, el origen y uso de los recursos, así como de los impactos de sus actividades a nivel ambiental y social, aparte de la calidad del producto o servicio.

Una manera de generar valor hacia los diferentes grupos de interés es mediante la adopción e implementación de modelos y programas de responsabilidad social 
empresarial (RSE, en adelante); estos modelos están encaminados a que las diferentes organizaciones tanto gubernamentales como empresariales aporten al mejoramiento social o ambiental y a una búsqueda simultánea de prosperidad económica, calidad ambiental y equidad social; por lo que en ocasiones se les tilda de iniciativas superficiales y orientadas más hacia mejorar las relaciones públicas y la reputación de las empresas y menos hacia un auténtico cambio en la forma en que se manejan las operaciones centrales del negocio (Guerrero, D. E. y Sandoval, J. H. 2011).

Sin embargo, existe evidencia actual de que la RSE se ha constituido en una preocupación y prioridad real para algunos empresarios y directivos, al demostrarse que la implementación de este tipo de estrategias está directamente relacionada con el desarrollo de las empresas, ya que los mercados de capitales en el mundo premian o castigan el manejo del medio en que ellas se desarrollan (Solano, 2005). Es decir que la operación ya no está ligada de manera exclusiva a generar rentabilidad (Friedman, M., 1970, y Carr, 1996) o empleo y riqueza (Palavecino, 2007); sino que debe estar articulada a un plan de gestión que le permita a cada empresa diferenciarse de los competidores además de contribuir a la sociedad (Porter \& Kramer, 2002, y Caravedo, B., 1998) y ser sustentable a largo plazo (Cajiga, 2012).

Según Fernando Olivares en su artículo "La acción social corporativa y el marketing con causa en España” (Fernando Olivares, 2000), plantea que uno de los valores que se ha visto en auge en la sociedad durante los últimos años es, entre otros, la solidaridad, y las empresas han tratado de obtener beneficio de ello mediante campañas de acción social o mercadeo con causa; por consiguiente, para lograr una mejora en su imagen corporativa, las empresas cada vez tratan de involucrarse más en temas de interés social o problemáticas de minorías (mujeres, ancianos, enfermedades, medio ambiente, Tercer Mundo).

Dicho de otro modo, la RSE empresarial es el compromiso de la empresa para con estas problemáticas, y las acciones sociales corporativas son todos aquellos actos que se supone van concorde a esta responsabilidad. Por ejemplo, campañas de alfabetización, donaciones públicas a diferentes ONG, reconstrucción de obras públicas.

Las empresas deben evaluar su rentabilidad, las condiciones legales a las cuales se deben acoger para desempeñar su actividad económica, integrarse voluntariamente al desarrollo sostenible de las comunidades, tener un manejo y cuidado ambiental. Es fundamental que las organizaciones identifiquen los focos de su responsabilidad y la capacidad que posee para cada una de sus prácticas de RSE, a su vez integrar estos procesos dentro de su cadena de valor, comunicarla con sus colaboradores, "transmitir de forma sistemática hacia el interior y exterior de la organización" (Porter, Las fuerzas de la economía, 2012, p. 24). Un instrumento por medio del cual es posible mostrar las acciones que se ejecutan en el modelo de RSE de una organización son los informes de sostenibilidad, ya que con ellos es posible mostrar los resultados de una manera estandarizada y sostenible, además de que permiten incluir los tres grandes componentes que también son considerados en la RSE (el económico, el ambiental y el social). Así mismo es posible mostrar el desempeño y 
comportamiento de la empresa y examinar cómo la RSE está en concordancia con las expectativas de los grupos de interés.

\section{Tributación y rSe en Colombia}

En Colombia el tema de la RSE es incipiente y aunque se ve reglamentado desde el año 1993, es muy poco lo que las organizaciones tanto gubernamentales como empresariales le han apuntado a manejar. El Centro Colombiano de Responsabilidad Empresarial (CCRE) considera que "la RSE es la forma de gestionar una organización mediante la interacción y relación armónica con sus diferentes grupos de interés dando respuesta a sus expectativas financieras, sociales y ambientales de manera que se contribuya al desarrollo sostenible y a la creación de valor en todo el sistema” (María, I., 2014).

Así mismo, las organizaciones no tienen un parámetro frente a los informes que se pueden presentar al momento de aplicar la RSE y al mismo tiempo los beneficios que se pueden obtener al ser socialmente responsable; el Global Reporting Initiative, más conocido como el GRI, o el Ethos, son informes desarrollados en aquellas organizaciones que han implementado la RSE y buscan a través de estos ser totalmente transparentes frente a los tres sectores: ambiental, económico y social. El GRI en su cuarta versión establece estándares para la revelación de las memorias de sostenibilidad que desarrollan las empresas y es una guía para la toma de decisiones al visualizar posibles riesgos.

En Colombia aquellas empresas que implementen dentro de sus planes de gestión empresarial la aplicación de la RSE tendrán beneficios y descuentos tributarios permitiendo de esta manera la realización de una planeación tributaria y manejo de los flujos de efectivo y optimización de los recursos.

A través del tiempo los tributos han ejercido influencia para generar beneficios que favorezcan las empresas y al Estado cuando se aplica la RSE; en el año 2012 con la reforma tributaria se crea el impuesto sobre la renta para la equidad (CREE), con el fin de que las empresas y sociedades aporten al Instituto Colombiano de Bienestar Familiar (ICBF) beneficiándose con la disminución de la carga prestacional en la contratación laboral. "La Ley establece asimetrías que atentan contra la libertad de oportunidades en el mercado” (Enciso, 2008, Diario La República). Por otro lado, los parámetros de la RSE y su aplicación en Colombia se han basado principalmente dentro de las normas de calidad iso 26000 (sistema de gestión de la responsabilidad social para las organizaciones) y la Iso 14000 (sistema de gestión ambiental para las organizaciones).

Cuando nos referimos a los tributos podemos decir que es la representación monetaria que realizan los contribuyentes, ya sean personas jurídicas o naturales, los cuales son de carácter obligatorio e impuestos por el Estado; estos son cuatro grupos:

- Impuestos: prestación en dinero o especie (bonos) que permiten alimentar el presupuesto de la Nación. 
- Aporte: pago con un beneficio específico y afecta solo a determinado grupo social y económico.

- Contribución: pago que se obtiene de la realización de las obras públicas o estatales y la destinación de estas solo se puede utilizar en la destinación para lo que fue creada.

- Tasa: cancelación de un servicio para el cual fue creado y se espera tener una retribución compensando el pago.

Los beneficios tributarios son estímulos establecidos por el gobierno con el fin de incentivar el desarrollo científico, tecnológico y la innovación; se reconocen como requisito fundamental para incentivar el crecimiento económico con el fin de mejorar la calidad de vida de sus ciudadanos. Es de tener en cuenta que los descuentos tributarios son valores aplicados al pago resultante de la liquidación del impuesto y se aplican aparte de los beneficios tributarios.

En el país no existen estudios que permitan dimensionar y cuantificar el conocimiento que tienen los profesionales encargados de implementar este tipo de políticas y programas. Es por esto que el presente trabajo busca una aproximación inicial, a través de un proyecto de investigación que planteó el objetivo de establecer el conocimiento que los profesionales de las áreas administrativas o contables tienen respecto a los beneficios tributarios que pueden aplicar al implementar un programa de RSE en sus respectivas empresas.

Este proyecto recobra importancia ya que dará una visión clara de los vacíos conceptuales que los empresarios o profesionales asociados a la toma de decisiones e implementación de programas a nivel administrativo en las empresas, tienen frente al tema de la RSE y plantear posibles soluciones.

\section{Metodología}

El proyecto de investigación se desarrolló a partir de la aplicación de dos encuestas de tipo cuantitativo/ cualitativo, aplicadas la primera a personal administrativo y personal del área contable y la segunda (mercadeo) a personal relacionado con el área en empresas de diferentes sectores en Colombia.

Las encuestas fueron desarrolladas a través de la plataforma Google Forms y enviadas vía correo electrónico para su diligenciamiento en línea. La tabulación de los datos se realizó a través del programa Excel 2007 y de la plataforma de Google Forms.

\section{Resultados}

Con el primer instrumento se logró encuestar un total de 38 personas del área administrativa/contable, de las cuales se reportaron 16 sectores productivos de la siguiente manera (figura 1 ): 
Figura 1. Distribución de los sectores productivos de las empresas encuestadas

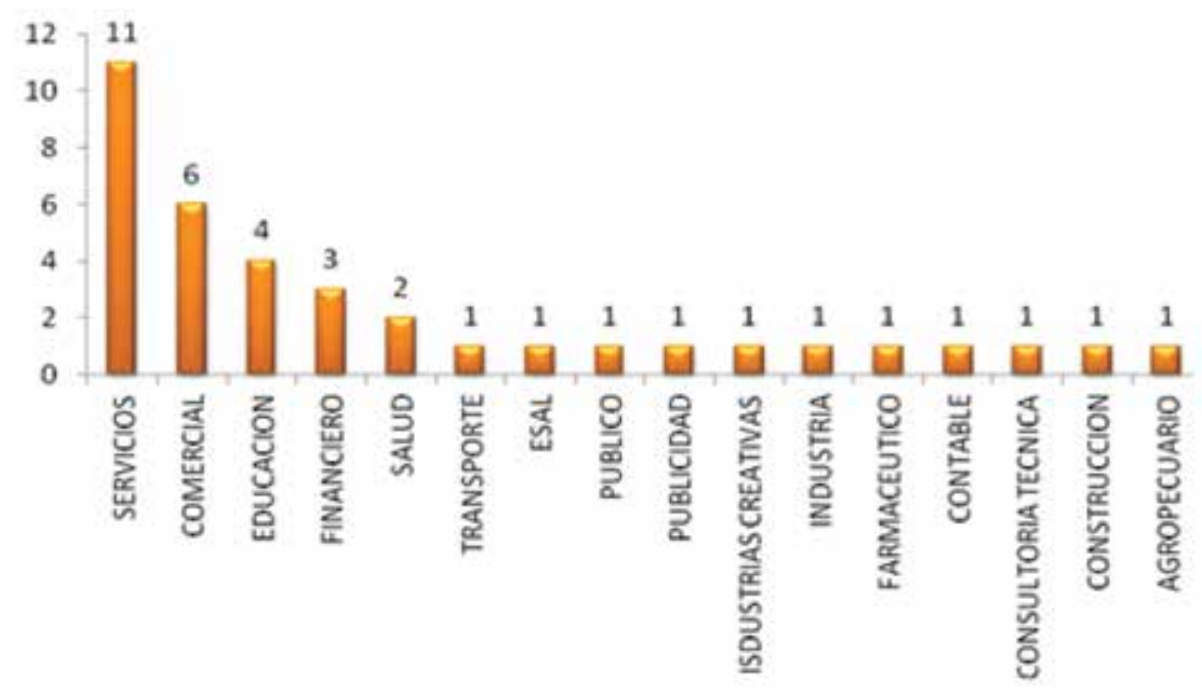

De las 38 personas encuestadas el 63,1\% corresponde al área contable y el 36,9\% al área administrativa (figura 2):

Figura 2. Área de desempeño laboral de las personas encuestadas

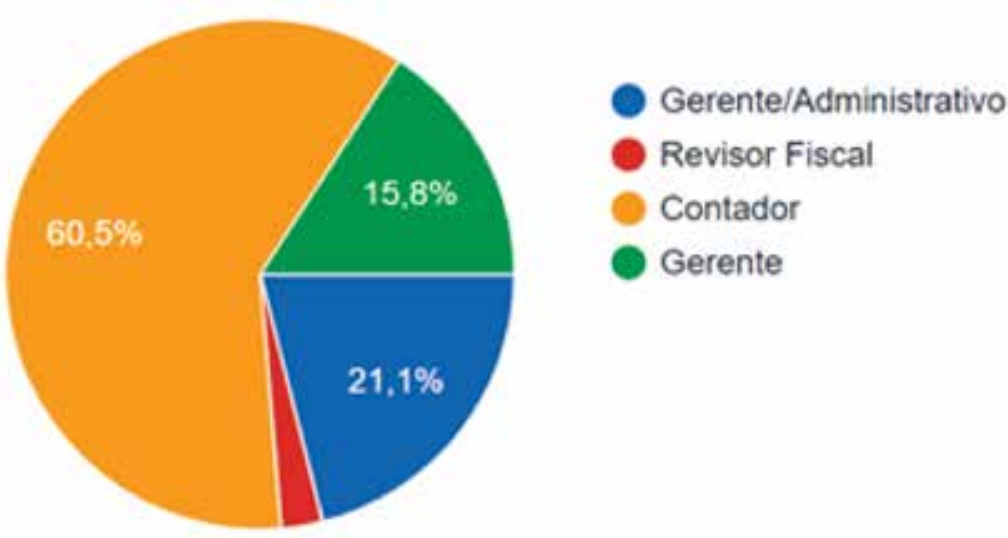

El 50\% de las personas encuestadas tienen una experiencia profesional de uno a tres años y solo el 2,6\% lleva más de diez años en su cargo (figura 3): 
Figura 3. Área de desempeño laboral de las personas encuestadas

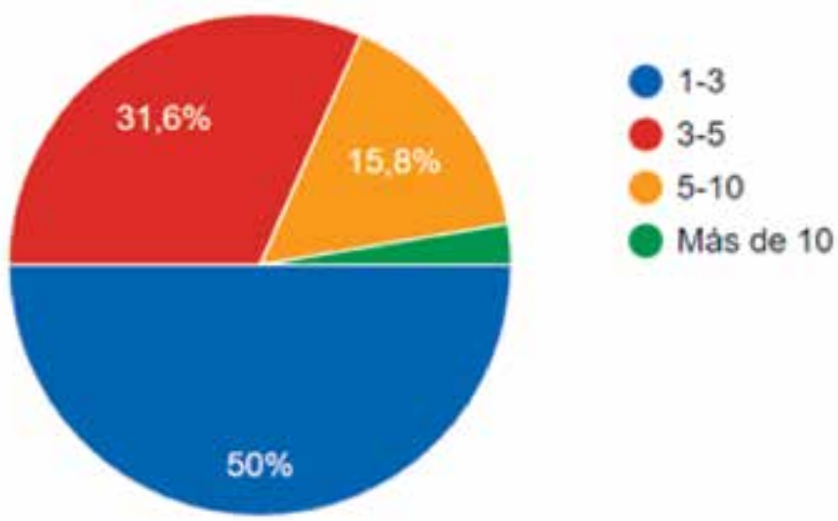

El 60,5\% de los encuestados reporta saber que es la RSE (figura 4), pero tan solo nueve de las 38 personas $(23,8 \%)$ conocen los ejes principales que conforman este tipo de programas y más de la mitad de los encuestados (52,6\%) afirma no tener ningún programa de RSE implementado en su empresa.

Figura 4. A. Conocimiento del concepto de RSE por parte de los encuestados. B. implementación de algún programa de RSE en la empresa

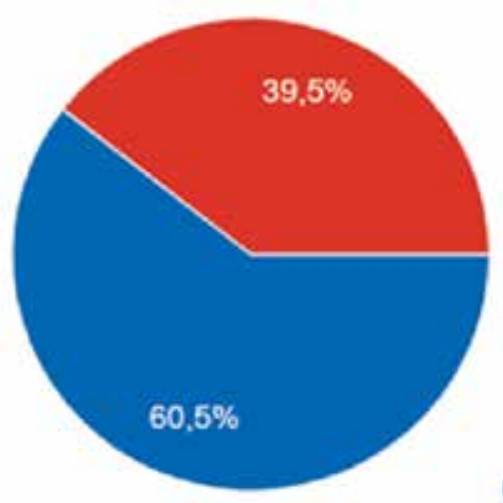

A

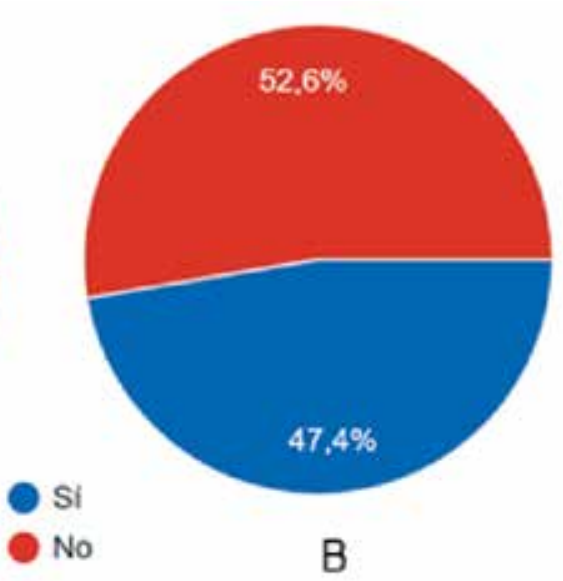

El 63,2\% de los encuestados reporta no saber a qué beneficios tributarios puede acceder la empresa al implementar un programa de RSE (figura 5) y los que sí conocen algún beneficio reportan: descuentos en la declaración de renta (cuatro personas), 
benéficos (no especificados) por contratación de personal en situación de discapacidad, deducciones y descuentos sin especificar y retribuciones por programas de reforestación.

Figura 5. Conocimiento de beneficios tributarios por implementación de programas de RSE por parte de los encuestados

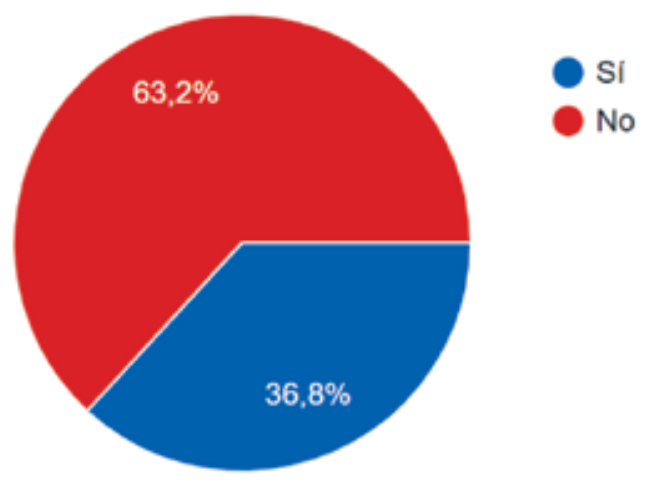

El 44,7\% afirma no conocer información sobre los beneficios tributarios a los que pueden aplicar al implementar programas de RSE y solo el $28,9 \%$ reporta haber recibido esta información en la universidad (figura 6).

Figura 6. A. Reporte de donde adquirió conocimiento sobre beneficios tributarios por implementación de programas de RSE por parte de los encuestados. B. Conocimiento sobre deducciones y descuentos tributarios relacionados específicamente con el eje social de la RSE
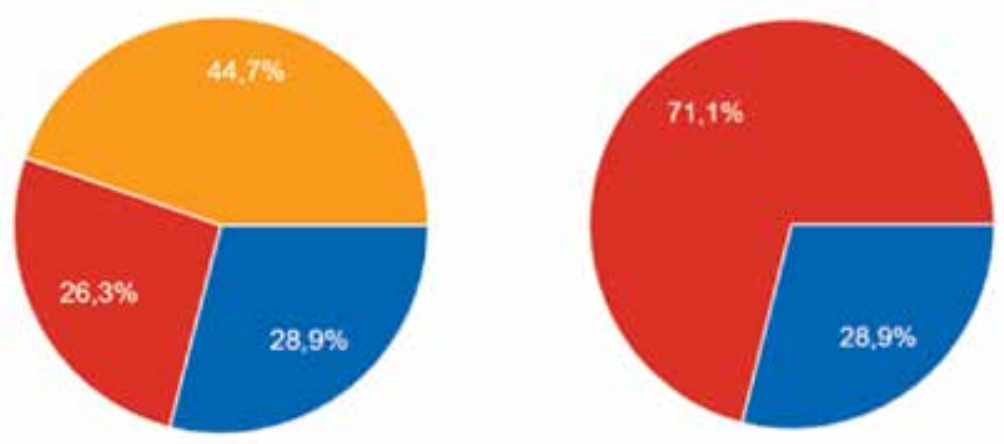

- En la universidad

En el ejercicio laboral

No conocia esta información

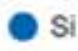


De los encuestados que afirman conocer los descuentos y deducciones específicos que reportan para el eje social: $100 \%$ deducibles a planes culturales relacionados con la lista representativa de patrimonio cultural internacional, donaciones, deducciones por inversiones a planes comunales de beneficios compartidos y beneficios por descuentos tributarios.

En cuanto al eje ambiental reportan: 100\% deducible a planes especiales de salvaguarda, reforestación, inversiones en plantaciones, riegos, buenas prácticas medioambientales (sin especificar qué beneficio o deducción) y deducción en renta.

En el segundo instrumento se encuestó a 42 personas, y los resultados arrojaron la siguiente información.

En cuanto a la relevancia de la RSE en el mercadeo y en la gestión de los stakeholders, los resultados del instrumento arrojaron resultados claros, pues el 78\% de los encuestados trabaja en empresas y el $92 \%$ considera que el término de responsabilidad social empresarial se define como "generar mejores condiciones en los grupos de interés (quienes puedan ser afectados o beneficiados por las actividades de una empresa)", es decir que reconocen le importancia de enfocar las labores socialmente responsables en focos que impulsen el desarrollo de la organización.

Por otra parte, al preguntar "¿quién cree usted que debería ser el principal agente socialmente responsable?”, el $44 \%$ de los encuestados respondió que el principal agente deben ser las empresas; sin embargo, el $41 \%$ asegura que el agente debe ser el Estado, siendo coherente con el mecanismo de organización que une las acciones entre la empresa, el Estado y la comunidad.

Los encuestados determinaron que el orden de importancia para crear un impacto positivo desde la RSE debe ser el siguiente: medio ambiente, comunidad, empleados, proveedores, empresarios, determinando el medio ambiente y la comunidad como los factores que determinan el éxito de la gestión de RSE en los stakeholders.

Paradójicamente, al preguntar sobre los obstáculos que existen para incorporar una estrategia de RSE en una empresa, el $41 \%$ considera que el principal obstáculo es la falta de compromiso de la empresa con la comunidad, y el $22 \%$ considera que el obstáculo es la falta de recursos.

Cuando se ahonda en si existe algún tipo preferencia por alguna empresa o producto que evidencie su influencia en la práctica de RSE, el 52\% de los encuestados reconoce que prefiere las marcas que son socialmente responsables, es decir que esta práctica es un factor fundamental en el mercadeo y en la gestión de los stakeholders; esta pregunta se complementa al determinar que un agente consumidor puede influir en la manera como una empresa es socialmente responsable, el $92 \%$ de los encuestados dicen que ellos podrían influir y la estrategia es recomendar esas empresas, esta respuesta tuvo un 72\% de acogida. El 93\% de los encuestados consideran que las empresas deben publicar sus acciones socialmente responsables a la sociedad, de esta forma el mercadeo influye en la decisión de compra. 


\section{Discusión}

Teniendo en cuenta la importancia que la implementación de programas de RSE les puede otorgar a las empresas y los valores agregados que representa, no solo en la fidelización de los diferentes grupos de interés, sino en términos de aportes sociales, ambientales, organizacionales y tributarios, solo el 47,4\% de las empresas encuestadas implementa algún programa de RSE.

El estudio se enfocó en personal del área administrativa y contable, teniendo en cuenta que estos perfiles laborales son los encargados del desarrollo e implementación de políticas, planes y programas en las empresas; así como del asesoramiento en temas contables y tributarios. Los hallazgos de este estudio demuestran una falta de conocimiento específico del tema, con el 63,2\% de los encuestados (la mayoría del área contable) que afirma no saber a qué beneficios tributarios puede acceder la empresa al implementar un programa de RSE.

Estos resultados resultan preocupantes, en un momento en el que Colombia debe cumplir unas metas ante la OCDE y otros compromisos internacionales adquiridos por acuerdos comerciales y tratados internacionales, en los cuales los países están esperando de las empresas la adopción e implementación de este tipo de programas como parte estructural de sus planes de gestión.

La mitad de los encuestados (50\%) se ha incorporado al ámbito laboral recientemente (entre uno y tres años) y solo el $28,9 \%$ de estos reporta obtener el conocimiento sobre la RSE en la universidad, lo cual plantea varia preguntas: ¿El desconocimiento sobre los temas de RSE se está dando por falta de preparación específica en la academia?, ¿Hace cuánto se vienen implementado los temas relacionados con RSE en las carreras contables y del área administrativa?, ¿Tienen todos los programas universitarios de estas área incorporado en el pénsum los temas de RSE?, ¿Actualmente existe una oferta adecuada de actualización académica para los profesionales que durante su proceso de formación universitaria no abordaron estos temas por ser relativamente nuevos?

Los resultados obtenidos en el presente estudio permiten evidenciar una tendencia de deficiencia en el conocimiento acerca de los beneficios y deducciones en aspectos tributarios y falta de conocimiento a profundidad sobre la RSE.

Al hacer un análisis de los datos cualitativos, se observa que las respuestas son ambiguas, sin especificidad, de carácter general y asociadas en su mayoría a un solo tipo de deducción (el de renta), lo cual demuestra que los encuestados que afirman conocer los beneficios y deducciones tienen un conocimiento muy somero del tema y desconocen de fondo el objetivo fundamental de este tipo normas.

Hoy en día las organizaciones dentro de las estrategias de planeación para los procesos de inversión incluyen la planeación tributaria, con el fin de establecer las metas de los montos a cancelar por estos conceptos; toma gran importancia lo que se conoce como "contabilidad de gestión", que "se considera uno de los instrumentos más utilizados como sistema informativo para la dirección, ya que permite conocer el resultado de la empresa y de cada una de sus áreas, contribuye de manera considerable a la formulación de objetivos y programas de operaciones, a la comparación 
del desempeño real con el esperado y a la realización de informes, ayudando así al proceso de toma de decisiones de una manera rápida y eficaz" (López, 2010).

La contabilidad de gestión vincula además la contabilidad de gestión ambiental, la cual está directamente relacionada con la implementación de los planes de RSE en las empresas, la cual "se refiere a la utilización de datos sobre los costos ambientales para la toma de decisiones en relación con el cuidado y protección del medio ambiente" (Isaac, D., \& Cañizares, M. 2019).

El Estado colombiano en su legislación ha establecido descuentos y deducciones tributarios para aquellas empresas que realicen inversiones no solo en el área ambiental, sino en apoyo al deporte, la investigación y desarrollo tecnológico e innovación, donaciones a entidades sin ánimo de lucro y empresas de "economía naranja”.

La gestión de los grupos de interés o stakeholders se sitúa como un elemento primordial para la creación de estrategias orientadas a la RSE en cualquier organización.

No se desconoce que el concepto de stakeholders se vincula siempre a las relaciones que la empresa establece con algunos de los agentes económicos más directos y más cercanos, tales como empleados, inversores, clientes o proveedores; sin embargo, esta concepción ha evolucionado en gran medida, pues se desarrollan modelos de producción entre las relaciones económico-productivas que se establecen en gran medida a la era industrial y postindustrial.

Siendo así, se puede indicar que es necesario y relevante crear mecanismos que planteen una relación clara y objetiva entre los agentes clave y la organización, para generar valor en el desarrollo de la actividad productiva de la misma.

Cabe resaltar que en el año 1983 se empiezan a analizar las relaciones preexistentes desde una perspectiva estratégica, "entendiéndolas como un ámbito clave para la consolidación y el éxito de las organizaciones” (Freeman, E., 1983). Esta visión presenta por primera vez a estas relaciones desde un marco estratégico, que amplía el foco corporativo, antes situado exclusivamente sobre los propietarios o accionistas de la organización (shareholders), a todos aquellos grupos que se ven afectados o pueden afectar el desarrollo de la actividad de la organización (stakeholders).

Es esta, sin duda alguna, una diferencia crucial que representa el cambio de paradigma, pues no era suficiente centrarse en las expectativas y demandas de aquellos grupos, inversores, accionistas o propietarios, que controlan la organización, sino que también es necesario tener en cuenta las necesidades, expectativas y demandas de otros grupos estrechamente vinculados a la empresa y que anteriormente sólo eran tenidos en cuenta de manera tangencial.

La presión que ejercen los grupos de interés a las empresas obliga a incorporar progresivamente principios de RSE en sus actividades productivas y procesos de gestión, lo cual ha modificado gradualmente las condiciones del entorno en el que compiten las empresas. El sorpresivo aumento de la presión reguladora modifica las pautas de la demanda en los mercados siendo estos correlacionados en los criterios inversión de los mercados financieros (RSE la última innovación en management, 2010, p. 5). 
Las crecientes iniciativas dirigidas a establecer una serie de principios y códigos de conducta con el fin de estimular y orientar a las empresas en la adopción de criterios de RSE han sido promovidas por una gran variedad de instituciones como organismos de carácter internacional como la Organización de las Naciones Unidas (ONU), la Organización para la Cooperación y el Desarrollo (OCDE), la Unión Europea, gobiernos y organizaciones privadas con el fin de informar a los stakeholders los resultados conseguidos en materia de RSE, para que conozcan cómo afectan sus intereses y estrategias de gestión. Esto debido a que los consumidores cada vez incorporan comportamientos sociales, ambientales o de tipo ético en los procesos de selección de productos. Este fenómeno se conocido como "consumo responsable" (Cruz Álvarez, Jesús Gerardo, Rodríguez Martha del Pilar, 2012, p. 34). Este consumo se relaciona implícitamente con la modalidad de demandar productos naturales, orgánicos y con una historia de tradición de fondo. Otra variable se manifiesta en la tendencia a consumir bienes producidos de acuerdo con criterios de RSE: materias primas obtenidas mediante comercio justo, bajo empleo de recursos no renovables, condiciones laborales dignas, etcétera (CONE, 2015).

Se considera necesario analizar los documentos de la organización que definen sus objetivos y su razón de ser para relacionar el nivel de coherencia entre las acciones y la misión y visión estipulada en un comienzo. Es necesario que los colaboradores conozcan y compartan la misión y los valores de la empresa para así tener correlación entre lo dicho y lo hecho, las actividades deben estar fundamentadas en el desarrollo del capital humano ya que se puede considerar el principal activo dentro de un proyecto, a su vez vinculándolos a un programa de voluntariado formativo, relacionado con las actividades que el colaborador desempeña en la organización, de esta manera no se perderán los vínculos laborales.

Las prácticas responsables deben ser divulgadas con tres parámetros fundamentales:

1. El contenido de la información debe ser puntual sobre las actividades que se están desarrollando, relacionando la misión y valores corporativos con el proyecto de responsabilidad.

2. Los valores que se transmiten deben ser lo más parecido a lo que se trabaja actualmente, logrando sensibilizar a la sociedad sobre el problema que se está trabajando.

3. Transparencia como entidad que cubre un proceso de desarrollo social logrando la credibilidad y apoyo de la sociedad civil.

Los procesos de RSE implican el reconocimiento de los intereses de quienes son afectados por los fines empresariales. La definición clásica de Freeman (1984) plantea que los stakeholders son grupos o individuos que puede afectar o ser afectados por los propósitos de la empresa. En esta medida, entonces, la empresa existe en interacciones necesarias con diversos actores previos que le definen y configuran su existencia, tales como socios, colaboradores y empleados, proveedores, acreedores, Estado y comunidad (Lévinas, 2016). 
El interés social está cada día más presente entre los ciudadanos, empresas, gobiernos, entre otros. "De parte de los gobiernos, algunos intentan imponer pautas de desarrollo en las cuales el crecimiento económico se encuentre en sintonía con el desarrollo social" (Naciones Unidas, 2016). En el plano internacional, existen organismos dedicados a promover igualdad y respeto de derechos internacionales intentando orientar el comportamiento que deben seguir las empresas. Por el lado de los ciudadanos se constatan movimientos de protección de los consumidores y ambientalismo, entre otros.

Lo principal que debe considerar una organización para realizar una correcta gestión de la RSE es identificar las expectativas y demandas de los grupos de interés, así como la influencia que tienen los mismos en la estrategia de la empresa, este sería el secreto para la administración exitosa de la herramienta y así lograr un posicionamiento socialmente responsable.

Una apuesta estratégica del contexto socioeconómico actual, donde los valores como la transparencia, la comunicación y el diálogo son los elementos organizacionales diferenciales de una compañía, estos diferenciales, son cada vez más apreciados por los distintos agentes a la hora de potenciar la sostenibilidad a largo plazo de la organización.

En este marco, la RSE puede decirse que surge como respuesta a los diversos retos que tienen las organizaciones con respecto a la sostenibilidad, entendiéndola no solo desde un punto de vista de la viabilidad económica de la organización), sino también social y medioambiental.

Las organizaciones de esta nueva era deben apostarle a actuar de forma socialmente responsable, lo cual implica integrar de manera estrictamente voluntaria las preocupaciones de sus grupos de interés en sus operaciones económicas y comerciales, siendo así la principal tendencia de consumo.

El vínculo esencial entre RSE y gestión de los grupos de interés es inmanente, pues la RSE no puede existir si no se tienen en cuenta las necesidades relevantes de los grupos de interés; sin esta relación, las empresas no podrán tener un papel destacado a la hora de desarrollar la actividad de la organización o diseñar o implementar su modelo de negocio.

Por tal motivo, para facilitar un modelo de integración de la relación con los grupos de interés "existen distintos niveles de gestión modulable en función de diferentes variables estratégicas, tales como la importancia del grupo de interés, su voluntad de cooperación, su posibilidad de influir en o ser influidos por la actividad de la organización" (Freeman E., 1983, p. 120).

Desde el punto de vista de la estrategia y la RSE, los stakeholders son relevantes pues posibilitan el intervenir en la organización y los resultados de esta. Siendo así, se logra minimizar los riesgos existentes en el mercado pues se fortalece la ventaja competitiva; además, mejora el clima y la reputación de la organización, y también promueve el aprendizaje y la innovación. Se puede afirmar que el proceso de relación con los grupos de interés es esencial de los procesos de inteligencia estratégica de una 
organización, pues permite conocer permanentemente la información con la que orientan las estrategias de la organización, reforzando la efectividad de sus acciones.

Por ende, los pasos que debe seguir una organización para gestionar la RSE en los stakeholders deben ser los siguientes: identificar los grupos de interés, establecer un modelo de gestión con los grupos de interés, involucrar a los distintos departamentos de la organización, desde la alta dirección hasta administración, pasando por compras, mercadeo, recursos humanos, medio ambiente, finanzas o calidad, entre otros. Y, por último, cada organización debe contemplarse desde una perspectiva transversal, de tal manera que pueda identificar todos aquellos grupos que puedan afectar o verse afectados por el desarrollo de la actividad.

El mercadeo cumple un papel importante en la gestión de la RSE y los stakeholders. Se evidencian varios modelos de sistemas de información de marketing (SIM), los cuales son de tipo descriptivo y de tipo gráfico. Estos modelos tienen como finalidad la recolección, clasificación, análisis, valoración y distribución de datos para la toma de decisiones y propuestas a demandas de los stakeholders, "el análisis de las oportunidades de marketing, la búsqueda y selección de público objetivo y posicionamiento de la oferta, el diseño de estrategias, la planificación de programas y la organización, gestión y control del esfuerzo de mercadeo" (p. 27) Kotler y Keller (2006).

Para los sistemas de información de marketing se sugiere adoptar un enfoque evolutivo e iterativo en su desarrollo, de forma tal que la relación calidad-uso-satisfacciónbeneficios genere un círculo virtuoso donde la buena acogida de los desarrollos iniciales del sistema detone la incorporación de nuevas prestaciones (Gómez, L., 2018).

El Global Reporting Initiative (GRI) es una iniciativa que establece un marco voluntario para la gestión multi-stakeholder, que evalúa las incidencias económicas, sociales y ambientales de la actividad de las organizaciones, evaluando los siguientes aspectos:

1. Relación de grupos de interés que la organización ha incluido.

2. Base para la identificación y selección de grupos de interés con los que la organización se compromete.

3. Enfoques adoptados para la inclusión de los grupos de interés, incluidas la frecuencia de su participación por tipos y categoría de grupos de interés.

4. Principales preocupaciones y aspectos de interés que hayan surgido a través de la participación de los grupos de interés y la forma en la que ha respondido la organización a los mismos en la elaboración de la memoria.

\section{Conclusiones}

Los resultados obtenidos en el presente estudio permitieron responder la pregunta de investigación y presentan evidencia preliminar de la falta de conocimiento específico de los profesionales de las áreas administrativas o contables sobre los beneficios y deducciones a los que pueden acceder las empresas al implementar programas de RSE. 
Adicionalmente, los resultados permiten tener una evidencia cuantitativa de los vacíos conceptuales relacionados con el tema de la RSE y plantean un aporte al área de investigación, al establecer preguntas claves a ser resueltas desde la academia, en estos temas tan importantes y actuales, para aportar en la formación de profesionales competitivos para el desarrollo empresarial del país.

El desconocimiento de la normatividad vigente sobre los beneficios y deducciones a los que pueden acceder las empresas puede ser un factor que está acentuando la falta de implementación de programas de RSE en Colombia, por tanto, impidiendo que se cumplan los objetivos gubernamentales que buscan que a partir de dichos beneficios y deducciones las empresas tengan una motivación para aportar positivamente en la adopción de políticas de producción más limpia, apoyo a la investigación, mejora de los procesos productivos, disminución de la huella de carbono y mejora de las condiciones sociales de los entornos laborales directos e indirectos.

Sin lugar a duda, la gestión de los stakeholders es el eje central de cualquier estrategia de RSE. Aunque históricamente estas relaciones se han visto en conflicto y generan riesgo, es fundamental que prime la comunicación bidireccional, las organizaciones deben ser testigo de las ventajas de alinear su estrategia con las expectativas y experiencias de la sociedad. Esta apertura al diálogo se está viendo como una oportunidad de innovación, no solo para anticipar riesgos, sino también para generar nuevos productos y servicios o adaptarlos contribuyendo así a una respuesta rápida y bien enfocada a las necesidades de los consumidores y por tanto más competitiva.

Son muchas más las ventajas de iniciar este diálogo de forma estratégica y es por tanto fundamental ampliar el conocimiento de los diferentes profesionales encargados de la planificación, desarrollo y toma de decisiones en las empresas frente las RSE y las oportunidades que su implementación puede traer a las empresas, no solo desde el punto de vista financiero y tributario, sino de fidelización e impacto positivo en diferentes áreas.

\section{REFERENCIAS BIBLIOGRÁFICAS}

CONE. (2015). Informe CONE RSE.

Cruz A. J. G. y Rodríguez M. (2012). Gestión de responsabilidad social. International Journal of Good Conscience.

Isaac, D., \& Cañizares, M. (2019). ¿Cómo vincular la información que brinda la contabilidad de gestión ambiental con los proyectos de inversión? Apuntes Contables, 9-23. doi: https://doi.org/10.18601/16577175.n23.02.

Freeman E. (1984). Los stakeholders en la RSE.

Freeman E. (1983). Strategic Management: A stakeholder Approach. Boston: Pitman. ISBN 0273-01913-9.

Lévinas, E. (2016). Aportes de la ética de Emmanuel Lévinas para el concepto de responsabilidad social empresarial.

Elósegui I. M. (2014). Libro verde para la RsC de la Comisión Europea. Zaragoza: European Framework for CSR. 
Naciones Unidas, K. A. (2016). Foro Económico Mundial (Suiza).

Olivares, F. (2000). La acción social corporativa y el marketing con causa en España. Revista de Estudios de Comunicación Komunikazio Ikasketen Aldizkaria, 9. Recuperado de http://www.ehu.eus/ojs/index.php/Zer/article/view/17434/15209.

Porter. (2006). Las fuerzas de la economía. Harvard Business Review.

Cajiga, J. El concepto de responsabilidad social empresarial. México: Centro Mexicano para la filantropía s/f.

Caravedo, B. (1998). Empresas responsables. Lima: sAsE y Perú.

Carr, A. (1996). Is business bluffing ethical? En: Rae, S.B. y Wong, K. L. (eds.).

Friedman, M. (1970). The Social Responsibility of business is to increase 1 ts profits. Times Magazine.

Gómez, L. (2018). Proposiciones teóricas de base para una concepción de Sistemas de información de Marketing. Apuntes Contables, 9-21. doi: https://doi.org/10.18601/16577175. n21.02.

Kotler y Keller (2006). Dirección de Marketing (12. a ed.). México: Pearson Educación. López, M. (2010). Sistemas de costo. La Habana: Félix Varela.

Palavecino E. La responsabilidad social empresarial: una mirada universitaria (tesis maestría). Santiago, Chile: Universidad Alberto Hurtado, 2007.

Porter, M. \& Kramer, M. “The Competitive Advantage of Corporate Philanthropy”, en Harvard Business Review, 2002; 80(12), 56-69. 46.

RSE la última innovación en management (2010). Universia Business Review.

Solano, D. Jáuregüi K. y Centeno A. (2005). Responsabilidad social corporativa: qué se hace y qué debe hacerse. Cuadernos de Difusión, 18-19. Perú: Universidad Esan: recuperado de https://www.esan.edu.pe/publicaciones/cuadernos-de.../numero-18-19/. 